\title{
Qualitätsbeurteilung in der Krankenhaushygiene
}

\author{
Roland Schulze-Röbbecke
}



Roland SchulzeRöbbecke

\section{Die drei Qualitätsdimensionen in der gesundheitlichen Versorgung}

Im Jahre 1966 veröffentlichte der armenischstämmige Mediziner Arvedis Donabedian das nach ihm benannte Modell, welches den heute noch gültigen Rahmen zur Untersuchung und Bewertung der Qualität der Patientenversorgung liefert [1]. Nach dem Donabedian-

Modell sind zur Beurteilung relevanter Qualitätsmerkmale der Patientenversorgung drei Arten von Informationen notwendig, nämlich Informationen über Strukturen, Prozesse und Ergebnisse (s. Abb. 1).

In der Terminologie der Qualitätssicherung und des Qualitätsmanagements im Gesundheitswesen wird das Donabedian-Modell inzwischen weltweit verwendet. Begriffe wie „Strukturqualität“, „Prozessqualität“ und „Ergebnisqualität“" gehören in der deutschsprachigen Medizin inzwischen zum Allgemeinwissen. Auch der Gemeinsame Bundesausschuss (G-BA) beruft sich bei der Erfüllung seines gesetzlichen Auftrags des Qualitätsmanagements und der Qualitätssicherung in der vertragsärztlichen und stationären medizinischen Versorgung auf dieses Modell [2].

\begin{tabular}{|c|c|c|}
\hline Struktur & Prozess & Ergebnis \\
\hline $\begin{array}{l}\text { Rahmen, in dem die } \\
\text { Patientenversorgung } \\
\text { stattfindet } \\
\text { (z.B. Gebäude, } \\
\text { Personal, Finanzierung, } \\
\text { technische Ausstat- } \\
\text { tung) }\end{array}$ & $\begin{array}{l}\text { Maßnahmen und } \\
\text { organisatorische } \\
\text { Abläufe, aus denen die } \\
\text { Patientenversorgung } \\
\text { besteht (insbes. } \\
\text { Diagnostik, Therapie, } \\
\text { Prävention) }\end{array}$ & $\begin{array}{l}\text { Auswirkung der } \\
\text { Patientenversorgung } \\
\text { auf die Gesundheit der } \\
\text { Patienten } \\
\text { (z.B. Zufriedenheit, } \\
\text { Änderung d. Gesund- } \\
\text { heitszustands, } \\
\text { Komplikationen, Tod) }\end{array}$ \\
\hline
\end{tabular}

Abb.1 Grafische Darstellung des Qualitätsmodells nach Donabedian.

\section{Relevante Qualitätsmerkmale in der Krankenhaushygiene}

Wie auf andere medizinische Fachgebiete ist das Donabedian-Modell auch auf die Krankenhaushygiene anwendbar:

- Die Ergebnisqualität lässt sich in der Krankenhaushygiene entsprechend ihren in $\S 23$ (3) des deutschen Infektionsschutzgesetzes (IfSG) formulierten Aufgaben durch Ermittlung der Rate nosokomialer Infektionen (NI) beurteilen, die in einer Einrichtung erworben wurden, und durch Ermittlung von Ereignissen, bei denen multiresistente Krankheitserreger (MRE) zwischen Patienten einer Einrichtung übertragen wurden. Instrument zur Beurteilung der Ergebnisqualität ist somit die in §23 (4) IfSG geforderte Aufzeichnung und Bewertung (Surveillance) von NI und MRE.

- Die Prozessqualität entspricht in der Krankenhaushygiene dem Ausmaß und der Zuverlässigkeit, mit denen Maßnahmen zum Erreichen der o.g. Ergebnisse umgesetzt werden. Von Bedeutung sind hier insbesondere Maßnahmen mit nachgewiesenem Effekt auf die Reduktion der NI-Entstehung und MRE-Verbreitung. Solche „evidenzbasierten Maßnahmen“ sollten sich besonders gegen die häufigsten NI richten, also gegen nosokomiale Sepsen, Pneumonien, Wund-, Harnwegs- und Clostridium-difficile-Infektionen. Bei der Prävention der MRE-Verbreitung stehen die rationale Antibiotika-Anwendung und die Händehygiene als evidenzbasierte Maßnahmen im Vordergrund.

- Die Strukturqualität kann in der Krankenhaushygiene anhand der baulichen, personellen, finanziellen etc. Strukturen beurteilt werden, die die Durchführung der o.g. Prozesse und damit das Erreichen der o.g. Ergebnisse ermöglichen und erleichtern. Für die Qualitätsbeurteilung sind insbesondere solche Strukturen von Bedeutung, die einen nachgewiesenen Effekt auf die Prozess- und Ergebnisqualität haben. Nachgewiesen sind solche Effekte z.B. für die Ausstattung einer Einrichtung mit Personal und dessen fachliche Qualifikation. Weitere Beispiele für Strukturen mit 
nachgewiesenem Effekt sind die Ausstattung einer Einrichtung mit Händedesinfektionsmittelspendern und persönlicher Schutzausrüstung.

Die Qualität der Krankenhaushygiene einer Einrichtung ist umso besser, je mehr sie über relevante Strukturen verfügt, je besser effektive Präventionsmaßnahmen als relevante Prozesse umgesetzt werden und je erfolgreicher niedrige NI-Raten und MRE-Übertragungsraten als relevante Ergebnisse (engl. outcomes) erzielt werden.

Für die Einstufung der krankenhaushygienischen Relevanz bestimmter Strukturen und Prozesse ist spezialisiertes medizinisches Fachwissen erforderlich, welches gleichzeitig für eine valide Gefährdungsbeurteilung im Bereich der Krankenhaushygiene unabdingbar ist. Das Fachwissen umfasst u. a. solide Kenntnissen über Epidemiologie, Ätiologie, Pathogenese, Transmission, Risikofaktoren und Prävention der verschiedenen NI, nach $\S 23$ (3) IfSG auf jeweils aktuellem Stand der medizinischen Wissenschaft. Über dieses Fachwissen verfügen normalerweise Krankenhaushygieniker, d. h. Fachärzte für Hygiene oder Mikrobiologie bzw. klinische Fachärzte mit einer strukturierten, curricularen Zusatzqualifikation [3]. Andere Studiengänge, z. B. der Biologie, Pharmazie oder Ingenieurwissenschaften sowie nichtakademische Berufsausbildungen (eine Ausnahme bildet hier in gewissen Grenzen die Ausbildung zur Hygienefachkraft) vermitteln dieses Fachwissen nicht.

\section{Spekulative Qualitätsmerkmale in der Krankenhaushygiene}

In letzter Zeit ist immer wieder zu beobachten, dass zur Qualitätsbeurteilung „hygienischer“ Aspekte im Gesundheitswesen irrelevante Struktur- und Prozesskriterien verwendet werden und die Ergebnisqualität vollends ausgeklammert wird. Dies betrifft z. B. Bemühungen verschiedener Organisationen zur Zertifizierung von Qualitätsmanagement (QM)-Systemen, bei denen Kriterien wie z. B. bauliche Merkmale sowie Aspekte der Hausreinigung und Kleiderordnung in den Vordergrund gestellt werden, für deren Relevanz bezüglich NI- und MRE-Prävention (Ergebnisqualität) es keine ausreichenden wissenschaftlichen Erkenntnisse gibt. Solchen Fehlbeurteilungen liegen offensichtlich nicht nur mangelnde Fachkenntnisse zu Grunde sondern auch Unkenntnis über das eigentliche Wesen der Krankenhaushygiene. Denn wie in der modernen, wissenschaftlich fundierten, evidenzbasierten Medizin werden auch in der Krankenhaushygiene nicht Maßnahmen mit spekulativem Effekt bevorzugt sondern Maßnahmen, deren Effekt nachgewiesen ist, möglichst mit Hilfe guter klinischer Studien.

\section{Aus Prozessen werden Ergebnisse}

Eine nähere Betrachtung verdienen Dokumente, in denen Prozesse zu Ergebnissen gemacht werden. Besonders bei traditionellen „Hygiene“-Themen wie Flächenreinigung, Medizinprodukte-Aufbereitung und anderen technischen Maßnahmen ist diese Tendenz zu beobachten. Hier wird z. B. die erfolgreiche Entfernung von Fluoreszenzmarkierungen auf Oberflächen [4] oder von kulturell nachweisbaren Bakterien aus Endoskopen [5] als „Ergebnisqualität“ bezeichnet. Nach dem Donabedian-Modell handelt es sich hierbei allenfalls um Kriterien, mit denen sich die Prozessqualität beurteilen lässt, nicht aber die „Ergebnisqualität“. Auch wenn solche Dokumente z.T. nicht vorgeben, die Ergebnisqualität der Patientenversorgung zu behandeln oder sogar explizit z.B. von der „Ergebnisqualität der Reinigung“ sprechen, so bleibt der fundamentale Unterschied zwischen diesen beiden Arten von „Ergebnisqualität“ oft unbemerkt. Selbst bei QM-Experten führt dies immer wieder zu Missverständnissen und Verwechslungen.

Mit Hilfe von Kriterien wie Fluoreszenzmarkierungen auf Oberflächen, Bakterien in Endoskopen und Luftkeimen lässt sich die Qualität von Reinigungs-, Desinfektions- und anderen Prozessen beurteilen; Rückschlüsse auf die Ergebnisqualität der Patientenversorgung erlauben sie aber nur bedingt. Das lässt sich anhand folgender Beispiele zeigen:

- Endoskope, die nach den gesetzlichen Vorgaben und international anerkannten Standards aufbereitet wurden, führten dennoch zu MRE-Übertragungen und nosokomialen Infektionen, z.T. mit Todesfolge [6]. Mit anderen Worten: Der Aufbereitungsprozess war einwandfrei, nicht aber das Ergebnis bezüglich der Gesundheit der Patienten.

- Das Prozesskriterium „keimfreie Luft“ lässt sich bei Operationen lüftungstechnisch mit Hilfe von schwebstoffgefilterter Zuluft und turbulenzarmer Verdrängungsströmung erzielen (Raumklasse Ia nach DIN1946-4). Das entscheidende Ergebniskriterium „Vermeidung postoperativer Wundinfektionen“ wird in Operationsräumen der Raumklasse Ia jedoch signifikant seltener erreicht als in Operationsräumen mit turbulent ausströmender, schwebstoffgefilterter Zuluft (Raumklasse Ib) [7]. Macht man hier die Prozessqualität zur Ergebnisqualität, so schadet man den 
operierten Patienten, indem man sie erhöhten Infektionsrisiken aussetzt.

Schon Donabedian hat auf die Schwierigkeit hingewiesen, Kausalbeziehungen zwischen Strukturen, Prozessen und Ergebnissen herzustellen [8]. Die oben aufgeführten Beispiele zeigen, dass die Krankenhaushygiene hier keine Ausnahme macht und wie komplex es auch hier ist, aus Kriterien der Prozessqualität Rückschlüsse auf die Ergebnisqualität der Patientenversorgung zu ziehen, d. h. auf die Verminderung des Risikos der NIEntstehung und MRE-Verbreitung. Um die qualitative Aussagekraft der verschiedenen Struktur- und Prozesskriterien beurteilen zu können, ist - wie bereits oben erwähnt - spezialisiertes medizinisches Fachwissen erforderlich.

\section{Folgen der Pervertierung von Qualitätsbegriffen}

Die Pervertierung, d.h. die Verdrehung und Verfälschung der Qualitätsdimensionen, insbesondere die Erhöhung der Prozessqualität zur Ergebnisqualität, hat in der Krankenhaushygiene weitreichende Folgen für die Patientensicherheit.

Der Ausdruck „Hygiene“ wird im allgemeinen Sprachgebrauch mit „Sauberkeit und Keimfreit“ gleichgesetzt [9]. Entsprechend §23 IfSG ist es aber nicht das Ziel der Krankenhaushygiene, für Sauberkeit und Keimfreit im Krankenhaus zu sorgen. Ihr Ziel und das eigentliche Ergebnis ihrer Aktivitäten ist es vielmehr, die Entstehung nosokomialer Infektionen (nicht nur durch MRE!) und die Verbreitung multiresistenter Erreger mit Maßnahmen zu vermeiden, die dem aktuellen Stand der medizinischen Wissenschaft entsprechen. Sauberkeit und Keimfreiheit der Patientenumgebung sowie die Aufbereitung von Medizinprodukten haben nur einen geringen Anteil daran, diese Ziele zu erreichen. Indem man solche-z.T. spekulativen-Qualitätskriterien zum Ergebnis krankenhaushygienischer Aktivitäten erhöht, degradiert man das spezialisierte Fachpersonal, insbesondere Krankenhaushygieniker und Hygienefachkräfte, zu Dienstleistern für Fragen von Ordnung, Sauberkeit und Keimfreiheit.
Durch eine solche Einengung des Blickfeldes werden nicht nur Ressourcen verschwendet. Auch das Wohl der Patienten gerät damit aus dem Blickfeld, mit der ständigen Gefahr, durch Unterlassung wesentlicher Präventionsmaßnahmen den Patienten Schaden zuzufügen [10]. Arvedis Donabedian wäre bestürzt, denn seinen Vorstellungen von der Qualität der Patientenversorgung entsprechen diese Entwicklungen mit Sicherheit nicht.

\section{Literatur}

1 Donabedian A. Evaluating the quality of medical care. Milbank Q 2005; 83: 691 - 729. Reprinted from Milbank Mem Fund Q 1966; 44: 166-203

2 Gemeinsamer Bundesausschuss. Struktur-, Prozess-, Ergebnisqualitätsvorgaben. (letzter Zugriff: 09.11.2016) https:// www.g-ba.de/institution/themenschwerpunkte/qualitaetssicherung/einrichtungsintern/qualitaetsvorgaben/

3 Kommission für Krankenhaushygiene und Infektionsprävention beim Robert Koch-Institut. Personelle und organisatorische Voraussetzungen zur Prävention nosokomialer Infektionen. Bundesgesundheitsbl 2009; 52: 951-962

4 Hausemann A, Hofmann $\mathrm{H}$, Otto $\mathrm{U}$ et al. Flächenreinigung und -desinfektion im Krankenhaus - Struktur-, Prozess- und Ergebnisqualität, Ergebnisse der infektionshygienischen Überwachung in Frankfurt am Main, 2014. Bundesgesundheitsbl 2015; 58: 620-630

5 Kommission für Krankenhaushygiene und Infektionsprävention beim Robert Koch-Institut. Bundesinstitut für Arzneimittel und Medizinprodukte. Anforderungen an die Hygiene bei der Aufbereitung von Medizinprodukten. Bundesgesundheitsbl 2012; 55 : $1244-1310$

6 Rubin ZA, Murthy RK. Outbreaks associated with duodenoscopes: new challenges and controversies. Curr Opin Infect Dis 2016; 29: 407-414

7 Gastmeier P, Breier AC, Brandt C. Influence of laminar airflow on prosthetic joint infections: a systematic review. J Hosp Infect 2012; 81: $73-78$

8 Donabedian A. The quality of care, how can it be assessed? JAMA 1988; 260: $1743-1748$

9 Schulze-Röbbecke R. Was ist Hygiene? Krankenhaushygiene up2date 2007; 2: 1-4

10 Schulze-Röbbecke R. Primum nocere. Krankenhaushygiene up2date 2010; 5: 73-74 